\title{
Inhibitor of DNA binding 4 functions as a tumor suppressor and is targetable by 5-aza-2'-deoxycytosine with potential therapeutic significance in Burkitt's lymphoma
}

\author{
XIAN-ZHENG GAO, WU-GAN ZHAO, GUAN-NAN WANG, MEI-YING CUI, YANG-RUI ZHANG and WEN-CAI LI \\ Department of Pathology, The First Affiliated Hospital of Zhengzhou University, Zhengzhou, Henan 450000, P.R. China
}

Received January 17, 2015; Accepted October 23, 2015

DOI: $10.3892 / \mathrm{mmr} .2015 .4640$

\begin{abstract}
Epigenetic gene silencing due to promoter methylation is observed in human neoplasia, including lymphoma and certain cancer types. One important target for gene methylation analysis in non-Hodgkin lymphoma (NHL) is inhibitor of DNA binding 4 (ID4). The present study aimed to investigate the gene methylation status of ID4, the expression of ID4 protein and the effect of demethylating agent 5-aza-2'-deoxycytosine (CdR) in the Raji human Burkitt's lymphoma cell line in vitro. Following assessment of the inhibition of Raji cell growth by various concentrations of $\mathrm{CdR}$, the effects of $\mathrm{CdR}$ on the expression of ID4 protein were assessed using the immunocytochemical streptavidin-peroxidase method and semi-quantitative analysis, while apoptosis and cell cycle were determined by flow cytometry. The ID4 gene methylation status of Raji cells was tested using methylation-specific polymerase chain reaction analysis. ID4 was methylated and its protein expression was low in the control group, while ID4 was partly or completely demethylated and its protein expression was upregulated in Raji cells treated with CdR. In addition, CdR induced apoptosis and cell cycle arrest in Raji cells in a dose- and time-dependent manner. These results demonstrated that ID4 is hypermethylated and its protein expression is low in Burkitt's lymphoma cells, while CdR reversed the abnormal DNA methylation and induced re-expression of ID4 protein. Hypermethylation of ID4 promotes the proliferation of Burkitt's lymphoma cells; ID4 may function as a tumor suppressor and can be targeted with demethylating compounds such as CdR for the treatment of Burkitt's lymphoma.
\end{abstract}

Correspondence to: Professor Wen-Cai Li, Department of Pathology, The First Affiliated Hospital of Zhengzhou University, 1 Jianshe East Road, Erqi, Zhengzhou, Henan 450000, P.R. China

E-mail: liwencaipatho@126.com

Key words: inhibitor of DNA binding 4, 5-aza-2'-deoxycytosine, Burkitt's lymphoma, Raji cells

\section{Introduction}

The transformation of normal stem cells into lymphoma is a multistep process comprising accumulated genetic and epigenetic aberrations, including mutations of the genome of proto-oncogenes, tumor suppressor genes and other genes associated with important cellular processes, such as differentiation and cell proliferation. Alterations in the methylation patterns of various genes have been observed in almost all cancer types, including hematological malignancies and solid tumors $(1,2)$. Numerous studies have shown that hypermethylation of $\mathrm{CpG}$ islands of tumor-suppressor genes, which are unmethylated under normal conditions, is associated with transcriptional silencing of the respective genes, which therefore has a critical role in tumor development and progression (1-4).

The inhibitor of DNA binding (ID) family is an important methylation site associated with non-Hodgkin lymphoma (NHL), which is utilized for clinical diagnosis. The functions of ID proteins comprise cell cycle control, lymphocyte development and cellular senescence $(5,6)$. By forming hetero-dimers with transcription factors, ID proteins act as dominant-negative inhibitors of gene transcription and negatively regulate the function of basic-helix-loop-helix (bHLH) transcription factors to affect the balance between cell growth and differentiation $(5,7)$. The ID protein family comprises four members, ID1-4, among which ID4 was first discovered in 2004 (8). However, its expression and function in various tumor types have remained controversial: Kuzontkoski et al (9) and Zeng et al (10) showed that ID4 is highly expressed in glioblastoma multiforme (GBM), in which it promotes angiogenesis and growth, while other studies showed that ID4 protein expression was decreased in several types of human cancer $(11,12)$. A study utilizing a mouse model of acute lymphoblastic leukemia of the T/natural killer cell lineage showed that ID4 protein expression was downregulated by promoter methylation and identified the ID4 gene as a putative tumor suppressor gene (8). The ID4 gene has also been confirmed to have an increased degree of methylation in a variety of human tumors $(13,14)$, including gastric adenocarcinoma (15), tumors of haematopoietic and lymphoid tissues $(8,16,17)$, breast carcinoma $(18,19)$, esophageal adenocarcinomas (20) and prostate cancers (12). ID4 methylation was also shown to be significantly correlated with World Health Organization sub-types and risk groups 
of cancer determined by the International Prognostic Scoring System. Multivariate analysis indicated that the ID4 methylation status was an independent prognostic factor for leukemia-free survival (21). Thus, the present study hypothesized that ID4 may be a therapeutic target in NHL. The demethylating reagent 5-aza-2'-deoxycytosine (CdR) inhibits DNA methyltransferases and reverses DNA methylation. It has been reported that CdR inhibits cancer cell growth, particularly that of leukemia cells. It has been applied for the treatment of myelodysplastic syndromes (MDS) and in preliminary experimental cancer treatments $(14,20,22)$, and the most clinically advanced agents, 5 -azacytidine and $\mathrm{CdR}$, provide a promising approach for the treatment of hematopoietic malignancies, including MDS and acute myeloid leukemia $(21,23)$. However, the mechanisms of action of CdR in NHL have largely remained elusive.

The present study explored the expression of ID4 protein and the status of ID4 gene methylation in the Raji human Burkitt's lymphoma cell line as well as their modulation by demethylating agent CdR. Furthermore, the effects of CdR on cell cycle distribution and apoptosis of Raji cells were investigated. The results demonstrated that ID4 was hypermethylated and its protein expression was low in Raji cells, while CdR reversed the abnormal DNA methylation and induced re-expression of the ID4 protein; furthermore, CdR enhanced apoptosis and induced cell-cycle arrest in Raji cells.

\section{Materials and methods}

Drugs and reagents. CdR was purchased from Sigma-Aldrich (St. Louis, MO, USA) and was dissolved in phosphate-buffered saline (PBS). ID4 rabbit polyclonal antibody (cat no. SC-B047) was purchased from Santa Cruz Biotechnology, Inc. (Dallas, TX, USA). One step modified base kit (cat. no. E112885) was purchased from Epigentek (Farmingdale, NY, USA). Ex Taq enzyme was purchased from Takara (Otsu, Japan).

Cell line and culture. The CCL-86 Raji human Burkitt's lymphoma cell line was purchased from the Chinese Academy of Sciences Shanghai Institute of Cell Biology (Shanghai, China) and was grown in RPMI-1640 culture medium (HyClone; GE Healthcare, Little Chalfont, UK)containing $10 \%$ fetal bovine serum, $100 \mathrm{U} / \mathrm{ml}$ penicillin and $100 \mathrm{mg} / \mathrm{ml}$ streptomycin (Harbin Pharmaceutical Group Holding Co., Harbin, China) in an incubator with a humidified atmosphere containing $5 \% \mathrm{CO}_{2}$ at $37^{\circ} \mathrm{C}$. Cells were passaged and inoculated at $2 \times 10^{5} / \mathrm{ml}$ in the logarithmic growth phase.

Cell growth study. Raji cells in the logarithmic growth phase were treated with $\mathrm{CdR}$ at $0,0.1,0.5,1.0,2.5$ or $5.0 \mu \mathrm{mol} / 1$. The total numbers of live cells in each group were counted every day for seven days under a light microscope and growth curves were drawn.

Treatment groups. On the basis of the growth curves, CdR at concentrations of $0,0.5$ and $5.0 \mu \mathrm{mol} / \mathrm{l}$ with incubation for 24,48 or $72 \mathrm{~h}$ were selected as the experimental conditions of the present study. In the control group, cells were treated with medium only. Cells were then subjected to analysis using the immunocytochemical streptavidin-peroxidase (SP) method,

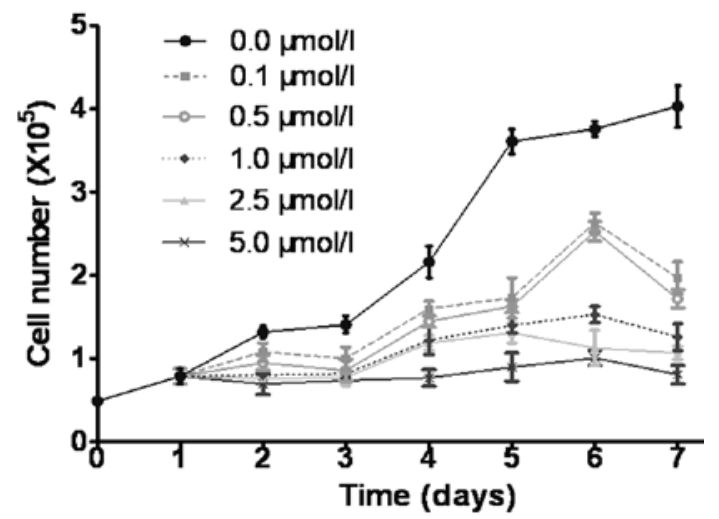

Figure 1. CdR inhibits the growth of Raji cells. Raji cells were divided into six groups that were treated with $\mathrm{CdR}$ at $0,0.1,0.5,1.0,2.5$ or $5.0 \mu \mathrm{mol} / 1$. The total number of live cells was then counted for seven consecutive days. CdR inhibited cell growth in a dose- and time-dependent manner. Values are expressed as the mean \pm standard deviation $(n=3)$. CdR, 5-aza-2'-deoxycytosine

flow cytometry and methylation-specific polymerase chain reaction (MS-PCR) technology to assess the expression of ID4 protein, apoptosis and cell cycle and the ID4 gene methylation status of Raji cells, respectively.

Immunocytochemical SP method and semiquantitative analysis of ID4 protein expression. Immunocytochemistry was performed according to a previously described method (24). Cells were seeded onto cover slips and peroxidase was blocked by incubating the cells with $3 \% \mathrm{H}_{2} \mathrm{O}_{2}$ (Maixin Biotech Co., Fuzhou, China) for $30 \mathrm{~min}$. Protein was blocked by incubation with normal goat serum (Maixin Biotech Co.) for $30 \mathrm{~min}$. Following incubation with ID4 rabbit polyclonal antibody at 1:100 dilution in PBS overnight at $4^{\circ} \mathrm{C}$, cells were treated with biotinylated secondary antibody (rat polyclonal; 1:50 dilution; cat. no. KIT-9710; Maixin Biotechnology) and peroxidase. Peroxidase-conjugated streptavidin was used with the Dako Real Detection System and diaminobenzidine (Dako, Glostrup, Denmark) according to the manufacturer's instructions. As a negative control, the primary antibody was replaced with PBS.

The intensity of ID4 staining in the cytoplasm was evaluated by two independent, experienced pathologists blinded to the experimental groups using a scale of 0-12. The correlation coefficient for the ID4 scoring determined by the two observers was $\mathrm{r}=0.93-0.96$.

Flow cytometric analysis. In each group, $1 \times 10^{6}$ Raji cells were harvested by centrifugation at $447 \mathrm{x} \mathrm{g}$, for $5 \mathrm{~min}$ and washed with PBS twice. The apoptosis assay was performed on Raji cells of each group using the Annexin V-FITC Apoptosis Detection Kit I (BD Biosciences, Franklin Lakes, NJ, USA) and analyzed by fluorescence-activated cell sorting.

For cell cycle analysis, cells were fixed by adding $700 \mathrm{ml} / \mathrm{l}$ ethanol dropwise on ice and incubation at $4{ }^{\circ} \mathrm{C}$ overnight. Following two washes with PBS, cells were incubated with propidium iodide (PI; Bender Medsystems, Vienna, Austria) for $20 \mathrm{~min}$ in the dark and analyzed by flow cytometry (FACSCalibur; BD Biosciences) with ModFit LT ${ }^{\mathrm{TM}}$ software (Verity Software House, Topsham, ME, USA) was used for quantification of cells in each phase of the cell cycle. 
A

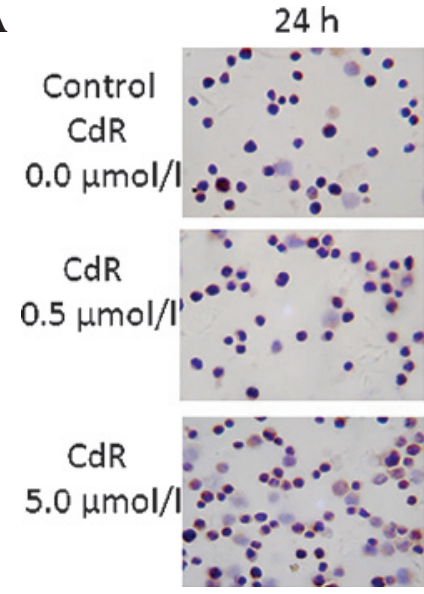

$48 \mathrm{~h}$

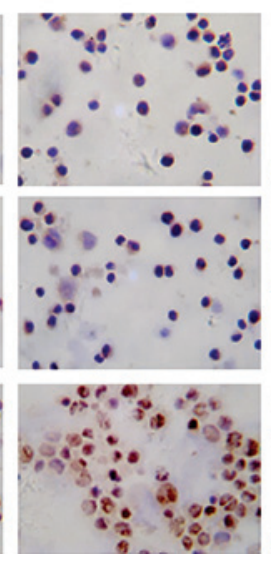

$72 \mathrm{~h}$

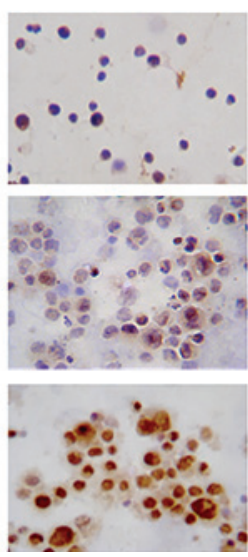

B

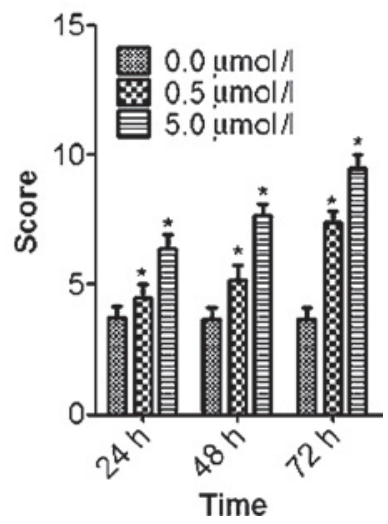

Figure 2. CdR upregulates the expression levels of ID4 protein. Raji cells were treated with CdR at final concentrations of $0,0.5$ or $5.0 \mu$ mol/1 for 24,48 or $72 \mathrm{~h}$. ID4 protein expression was examined using the immunocytochemical streptavidin-peroxidase method and semi-quantitative analysis. (A) Representative images showing the expression levels of ID4 protein in the treated and control groups (magnification, x400). (B) Semi-quantitative analysis of ID4 protein expression showed that ID4 was upregulated by CdR in a dose-and time-dependent manner. Values are expressed as the mean \pm standard deviation of three experiments. * $\mathrm{P}<0.05$ vs. control. CdR, 5-aza-2'-deoxycytosine; ID4, inhibitor of DNA binding 4.

MS-PCR. For MS-PCR, sodium bisulfite-treated DNA was amplified using either a methylation-specific or a non-methylation-specific primer set, synthesized by AuGCT Co. (Beijing, China). The sequences of the methylation-specific primers were 5'-TTTTATAAATATAGTTGCGCGGC-3' (forward) and 5'-GAA ACTCCGACTAAACCCGAT-3' (reverse). Sequences of the non-methylation-specific primers were 5'-GTTTTATAAATATAGTTGTGTGGTGG-3' (forward) and 5'-AAAACTCCAACTAAACCCAATCT-3' (reverse) (24). DNA extracted from the Raji cell line using the QlAamp DNA Mini kit (Qiagen, Hilden, Germany) and then methylated at $\mathrm{CpG}$ sites using $\mathrm{CpG}$ methylase enzyme (New England Biolabs, Ipswich, MA, USA) according to the manufacturer's instructions. DNA derived from the human breast cancer cell line MDA-MB231 (Chinese Academy of Sciences Shanghai Institute of Cell Biology) was used as a positive control, whose DNA was extracted using a procedure identical to that of the Raji cells, and distilled water was treated with bisulfide and CpG methylase to serve as a negative control (17). MSP was performed with the following cycling conditions: $95^{\circ} \mathrm{C}$ for $5 \mathrm{~min} ; 39$ cycles of denaturation at $95^{\circ} \mathrm{C}$ for $1 \mathrm{~min}$; specific annealing at $59^{\circ} \mathrm{C}$ for $1 \mathrm{~min}$ and extension for $72^{\circ} \mathrm{C}$ for $1 \mathrm{~min}$; and a final extension of $7 \mathrm{~min}$ at $72^{\circ} \mathrm{C}$. Takara $\mathrm{Taq}^{\mathrm{TM}} \mathrm{Hot}$ Start Version (Takara) was used in the experiment. The PCR mixture contained $50 \mathrm{ng}$ bisulfite-treated DNA, $4 \mu \mathrm{l}(2.5 \mathrm{mM})$ deoxynucleoside triphosphate mixture, $0.5 \mu \mathrm{l}(20 \mathrm{M})$ of each primer, 10X PCR buffer and 1.25 units of Takara Taq enzyme in a total volume of $50 \mu \mathrm{l}$. PCR was performed in a PTC-200 cycler (Bio-Rad Laboratories, Inc., Hercules, CA, USA). The amplification products were analyzed on $2.2 \%$ agarose gels with 50-bp DNA Ladder Maker (Takara) and visualized under ultraviolet illumination (Ultra-Violet Products Ltd., Cambridge, UK).

Statistical analysis. All experiments were repeated three times. All values are expressed as the mean \pm standard deviation and analyzed using SPSS 19.0 software (International Business Machines, Armonk, NY, USA). Significance of comparisons between experimental groups was tested using Student's t-test.
$\mathrm{P}<0.05$ was considered to indicate a statistically significant difference.

\section{Results}

CdR inhibits cell growth. The growth curves indicated that CdR inhibited the growth of Raji cells in a time- and dose-dependent manner (Fig. 1). As marked differences between the growth curves of cells treated with $0,0.5$ and $5.0 \mu \mathrm{mol} / 1 \mathrm{CdR}$ were present, these conditions were used in subsequent experiments.

CdR enhances the expression of ID4 protein. In untreated Raji cells, ID4 protein expression was low and only present in the cytoplasm. However, a marked increase in ID4 protein expression was observed following CdR treatment, and ID4 was present in the cytoplasm and in the nucleus (Fig. 2A). The expression of ID4 was highest after $72 \mathrm{~h}$ of incubation with $5.0 \mu \mathrm{mol} / \mathrm{l} \mathrm{CdR}$. CdR significantly increased the protein expression of ID4 in a concentration-dependent manner with constant incubation time $(\mathrm{P}<0.05)$; furthermore, $\mathrm{CdR}$ at a constant concentration of $5.0 \mu \mathrm{mol} / 1$ significantly increased the expression of ID4 in a time-dependent manner $(\mathrm{P}<0.05)$ (Fig. 2B).

$C d R$ causes apoptosis and $S$-phase arrest in Raji cells. The effects if CdR on the apoptotic rate and cell cycle of Raji cells were detected by flow cytometry. The number of apoptotic cells was significantly increased by $\mathrm{CdR}$ treatment in a concentration- and time-dependent manner $(\mathrm{P}<0.05)$ (Fig. 3). Furthermore, CdR significantly enhanced the S-phase population of Raji cells in a concentration- and time-dependent manner $(\mathrm{P}<0.05)$, indicating that $\mathrm{CdR}$ causes cell cycle arrest in $\mathrm{S}$ phase in Raji cells (Fig. 4).

$C d R$ reverses the hypermethylation of ID4 in Raji cells. Untreated Raji cells exhibited methylated ID4 only, indicating that ID4 is hypermethylated in Raji cells. Of note, in Raji cells treated with $5.0 \mu \mathrm{mol} / 1 \mathrm{CdR}$ for $72 \mathrm{~h}$, only unmethylated ID4 was present, indicating that $\mathrm{CdR}$ had the capacity to fully 
A

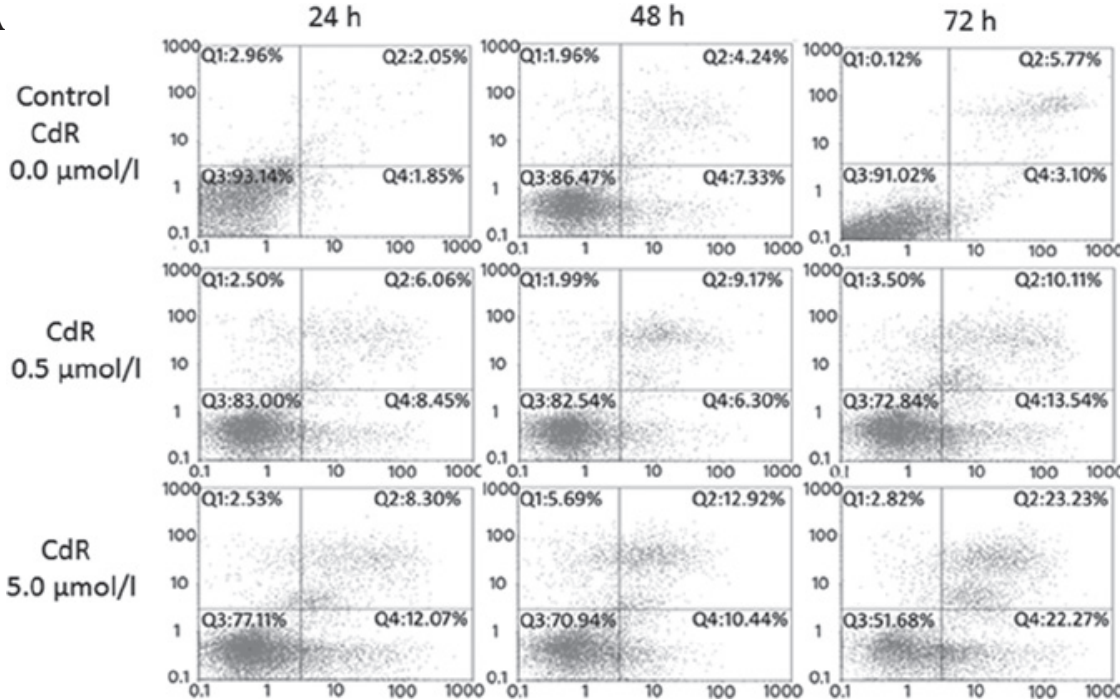

B

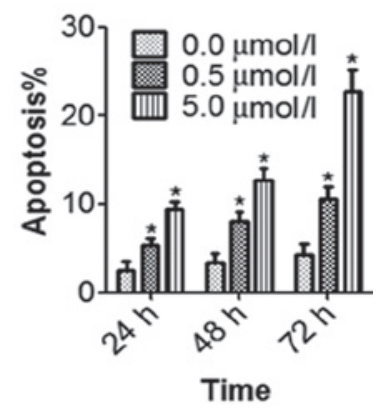

Figure 3. CdR promotes apoptosis of Raji cells. Raji cells were divided into three groups that were treated with CdR at 0, 0.5 or $5.0 \mu \mathrm{mol} / 1$. (A) Apoptosis was analyzed using flow cytometry following Annexin V (x-axis) and propidium iodide staining (y-axis). In the representative graphs, Q1, Q2, Q3 and Q4 resemble the populations of dead cells (no significance), apoptotic cells, live cells and cells in the pro-phase of apoptosis, respectively. (B) The ratio of apoptotic cells vs. total cells in each group shows that CdR promoted apoptosis of Raji cells in a time- and dose-dependent manner. Values are expressed as the mean \pm standard deviation of three experiments. " $\mathrm{P}<0.05$ vs. control. CdR, 5-aza-2'-deoxycytosine.

A

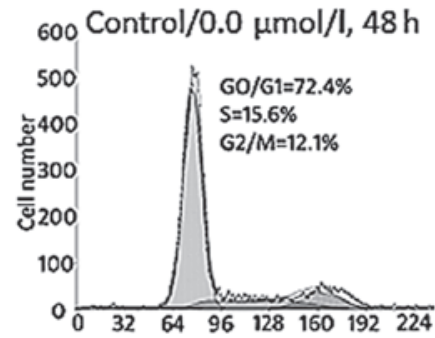

C

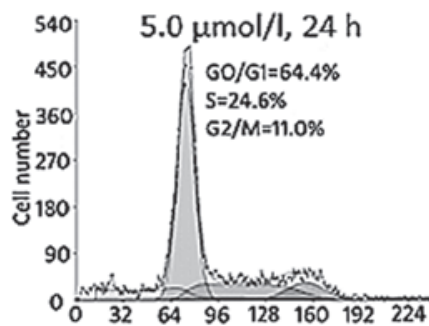

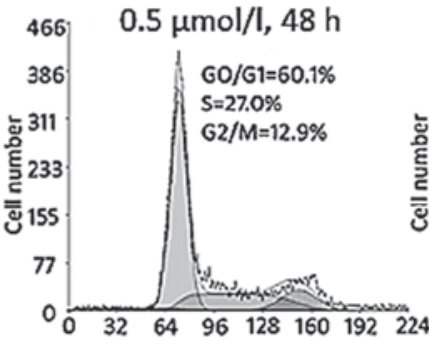
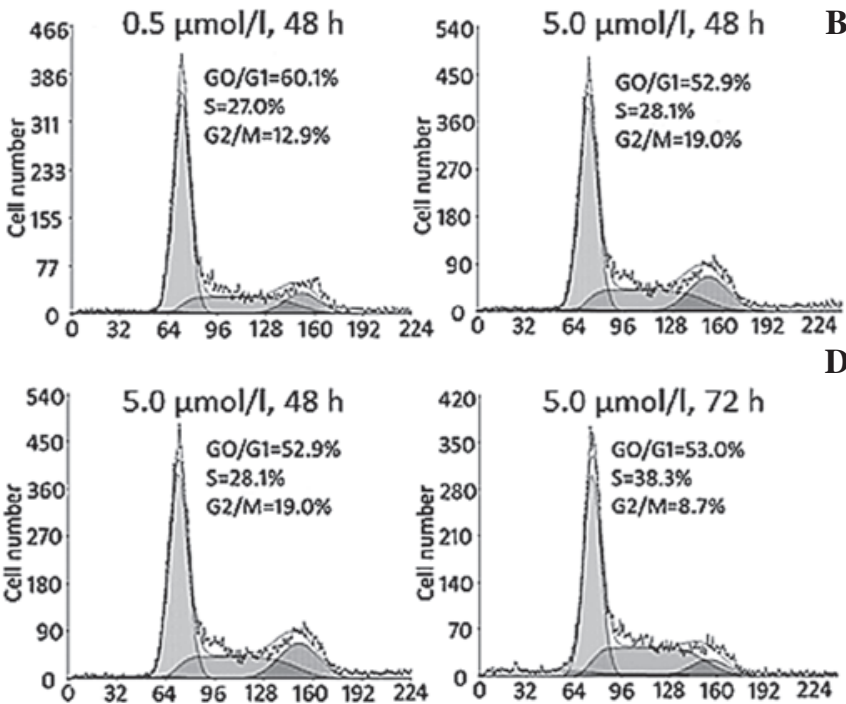

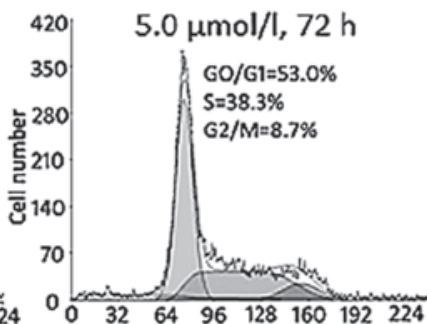

B

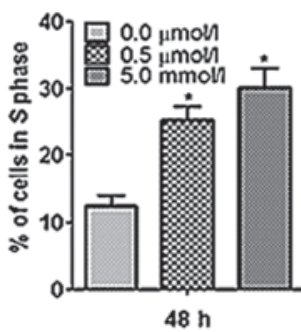

D

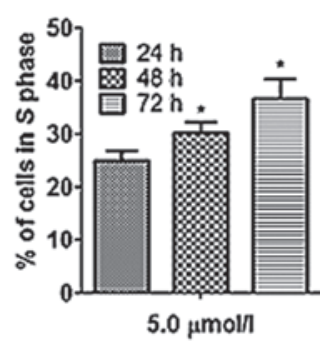

Figure 4. CdR causes cell cycle arrest in S phase in Raji cells in a time- and dose-dependent manner. CdR was used to treat Raji cells at final concentrations of $0,0.5$ or $5.0 \mu \mathrm{mol} / 1$ for 24,48 or $72 \mathrm{~h}$. Cell cycle analysis was performed by flow-cytometric analysis following staining with propidium iodide. (A) Cell cycle distribution of Raji cells in each CdR treatment group following incubation for $48 \mathrm{~h}$. (B) The ratio of cells in $\mathrm{S}$ phase from A. " $\mathrm{P}<0.05$ vs. control. (C) Cell cycle of Raji cells treated with $5.0 \mu \mathrm{mol} / 1 \mathrm{CdR}$ for 24,48 or $72 \mathrm{~h}$. (D) The ratio of cells in $\mathrm{S}$ phase from C. Values are expressed as the mean \pm standard deviation $(\mathrm{n}=3) .{ }^{*} \mathrm{P}<0.05$ vs. control. CdR, 5-aza-2'-deoxycytosine.
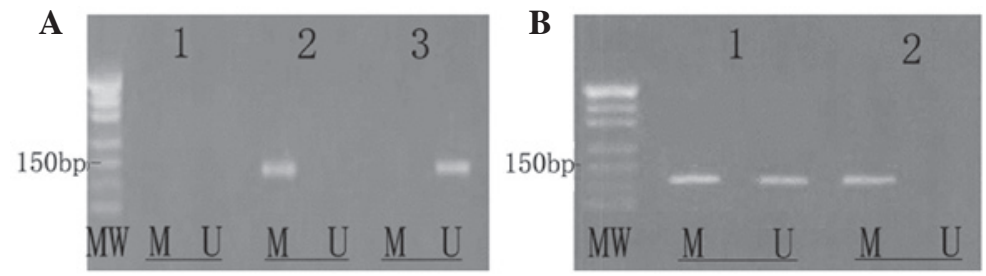

Figure 5. CdR reverses abnormal DNA methylation. Raji cells were treated with CdR $(0,0.5$ or $5.0 \mu \mathrm{mol} / 1)$ for 24,48 or $72 \mathrm{~h}$. Methylation-specific polymerase chain reaction was used to test the ID4 gene methylation status in each group. (A) Lanes: 1, blank control group (DNA replaced with distilled water); 2, Raji cells treated with medium only - the methylated gene as the sole amplification product indicated that the ID4 gene is completely methylated in Raji cells; 3, Raji cells treated with $5.0 \mu \mathrm{mol} / 1 \mathrm{CdR}$ for $72 \mathrm{~h}$ - amplification of the unmethylated gene only indicated that the ID4 gene was fully demethylated by CdR. (B) Lanes: 1, Raji cells treated with $5.0 \mu \mathrm{mol} / 1 \mathrm{CdR}$ for $48 \mathrm{~h}$ - amplification of methylated and unmethylated gene indicated that the ID4 gene was partially demethylated by CdR; 2, in untreated Raji cells - only the methylated gene was amplified. MW, molecular weight marker; M, methylated gene fragment; U, unmethylated gene fragment; CdR, 5-aza-2'-deoxycytosine; ID4, inhibitor of DNA binding 4. 
demethylate the gene. In the group treated with $0.5 \mu \mathrm{mol} / 1$ for $48 \mathrm{~h}$, methylated as well as unmethylated ID4 was present, indicating that the gene was partially methylated (Fig. 5).

\section{Discussion}

ID4 has become a hot spot in cancer research due to its heterogeneous roles in various cancer types (25). ID4 has been observed to be involved in human neoplasia, including lymphoma, GBM and breast cancer, and also represents a therapeutic target. However, as studies on the function of ID4 in various cancer types display inconsistencies, further clarification of its roles and the underlying mechanisms is required. ID4 protein has been reported to enhance tumor angiogenesis and to be is highly expressed in GBM $(10,26)$; however, ID4 protein expression was shown to be decreased in several types of human cancer $(7,11)$ and ID4 is a putative tumor suppressor gene $(8,26)$. Therefore, the present study was performed to identify the role of ID4 in Bukitt's lymphoma.

As ID4 has been suggested to be a crucial factor controlling cell differentiation, the present study hypothesized that epigenetic regulation of the ID4 gene may affect differentiation and progression of Burkitt's lymphoma. In the present study, the link between ID4 promoter hypermethylation, protein expression levels and apoptosis was determined.

First, immunocytochemistry and semi-quantitative analysis of ID4 protein expression proved that the expression of ID4 protein in Raji cells was inhibited, which was consistent with the results of previous studies $(2,23,24)$. The detection of ID4 protein may aid in the diagnosis of Burkitt's lymphoma, which requires confirmation by future study. In addition, ID4 protein was upregulated by $\mathrm{CdR}$ in a dose- and time-dependent manner. These results suggested that demethylation of the promoter region of ID4 silenced through hypermethylation in lymphoma may represent a novel treatment approach of high therapeutic value and good prospects.

Furthermore, flow cytometric analysis performed in the present study showed that CdR enhanced apoptosis in Raji cells and caused cell cycle arrest in $\mathrm{S}$ phase. To the best of our knowledge, the present study was the first to report that CdR induces cell apoptosis. It can be concluded that hypermethylated ID4 promoted the proliferation of Burkitt's lymphoma cells, which is in agreement with the results of $\mathrm{Qu}$ et al (27). Of note, CdR was able to demethylate ID4, which may be of therapeutic significance and improve the outcome of Burkitt's lymphoma.

The MS-PCR results of the present study were consistent with those of previous studies $(27,28)$, suggesting that specific methyltransferase inhibitor CdR reversed the abnormal DNA methylation and induced the re-expression of ID4 protein, thereby effectively inhibiting the proliferation and promoting the differentiation and apoptosis of NHL cells. Abnormal DNA methylation has also been shown to be reversed by other drugs, including arsenic trioxide (27); therefore, DNA demethylating agents may represent a novel clinical treatment approach. In general, the treatment of ID4 gene methylation in NHL is of great therapeutic significance.

In conclusion, the present study indicated that the ID4 gene was hypermethylated and its protein expression was low in Burkitt's lymphoma cells, while CdR reversed the abnormal DNA methylation and induced re-expression of the ID4 protein. Hypermethylation of tumor suppressor gene ID4 promotes the proliferation of Burkitt's lymphoma cells, which can be reversed by demethylating drug CdR, which represents a promising therapeutic approach for Burkitt's lymphoma.

\section{Acknowledgements}

The present study was supported by the National Natural Science Foundation of China (grant no. U1204814). The authors would like to thank the Henan Key Laboratory for Tumor Pathology (Department of Pathology, The First Affiliated Hospital of Zhengzhou University, Zhengzhou, China) for their technical assistance, including Miss Dong-Ling Gao and Miss Lan Zhang for assistance with flow cytometric analysis, Miss Shuang Xue for MS-PCR, as well as Dr Sheng-Lei Li and Miss Ming Huang.

\section{References}

1. Sandoval J, Mendez-Gonzalez J, Nadal E, Chen G, Carmona FJ, Sayols S, Moran S, Heyn H, Vizoso M, Gomez A, et al: A prognostic DNA methylation signature for stage I non-small-cell lung cancer. J Clin Oncol 31: 4140-4147, 2013.

2. Esteller M: Dormant hypermethylated tumour suppressor genes: Questions and answers. J Pathol 205: 172-180, 2005.

3. Sato T, Arai E, Kohno T, Tsuta K, Watanabe S, Soejima K, Betsuyaku T and Kanai Y: DNA methylation profiles at precancerous stages associated with recurrence of lung adenocarcinoma. PLoS One 8: 59444, 2013.

4. Guillaumet-Adkins A, Richter J, Odero MD, Sandoval J, Agirre X, Catala A, Esteller M, Prósper F, Calasanz MJ, Buño I, et al: Hypermethylation of the alternative AWT1 promoter in hematological malignancies is a highly specific marker for acute myeloid leukemias despite high expression levels. J Hematol Oncol 7: 4, 2014.

5. Zebedee Z and Hara E: Id proteins in cell cycle control and cellular senescence. Oncogene 20: 8317-8325, 2001.

6. Rivera $\mathrm{R}$ and Murre $\mathrm{C}$ : The regulation and function of the $\mathrm{Id}$ proteins in lymphocyte development. Oncogene 20: 8308-8316, 2001.

7. Yu L, Liu C, Vandeusen J, Becknell B, Dai Z, Wu YZ, Raval A, Liu TH, Ding W, Mao C, et al: Global assessment of promoter methylation in a mouse model of cancer identifies Id4 as a putative tumor-suppressor gene in human leukemia. Nat Genet 37: 265-274, 2005.

8. Wilson JW, Deed RW, Inoue T, Balzi M, Becciolini A, Faraoni P, Potten CS and Norton JD: Expression of Id helix-loop-helix proteins in colorectal adenocarcinoma correlates with p53 expression and mitotic index. Cancer Res 61: 8803-8810, 2001.

9. Kuzontkoski PM, Mulligan-Kehoe MJ, Harris BT and Israel MA: Inhibitor of DNA binding-4 promotes angiogenesis and growth of glioblastoma multiforme by elevating matrix GLA levels. Oncogene 29: 3793-3802, 2010.

10. Zeng W, Rushing EJ, Hartmann DP and Azumi N: Increased inhibitor of differentiation 4 (id4) expression in glioblastoma: A tissue microarray study. J Cancer 1: 1-5, 2010.

11. Arnold JM, Mok SC, Purdie D and Chenevix-Trench G: Decreased expression of the Id3 gene at 1p36.1 in ovarian adenocarcinomas. Br J Cancer 84: 352-359, 2001.

12. Deleu S, Savonet V, Behrends J, Dumont JE and Maenhaut C: Study of gene expression in thyrotropin-stimulated thyroid cells by cDNA expression array: ID3 transcription modulating factor as an early response protein and tumor marker in thyroid carcinomas. Exp Cell Res 279: 62-70, 2002.

13. Lasorella A, Uo T and Iavarone A: Id proteins at the cross-road of development and cancer. Oncogene 20: 8326-8333, 2001.

14. Hagiwara K, Nagai H, Li Y, Ohashi H, Hotta T and Saito H: Frequent DNA methylation but not mutation of the ID4 gene in malignant lymphoma. J Clin Exp Hematop 47: 15-18, 2007. 
15. Umetani N, Mori T, Koyanagi K, Shinozaki M, Kim J, Giuliano AE and Hoon DS: Aberrant hypermethylation of ID4 gene promoter region increases risk of lymph node metastasis in T1 breast cancer. Oncogene 24: 4721-4727, 2005.

16. Cen J, Shen J, Wang X, Kang H, Wang L, Sun L, Li Y and Yu L: Association between lymphoma prognosis and aberrant methylation of ID4 and ZO-1 in bone marrow and paraffin-embedded lymphoma tissues of treatment-naive patients. Oncol Rep 30: 455-461, 2013.

17. Smith E, De Young NJ, Pavey SJ, Hayward NK, Nancarrow DJ, Whiteman DC, Smithers BM, Ruszkiewicz AR, Clouston AD, Gotley DC, et al: Similarity of aberrant DNA methylation in Barrett's esophagus and esophageal adenocarcinoma. Mol Cancer 7: 75, 2008.

18. Noetzel E, Veeck J, Horn F, Hartmann A, Knüchel R and Dahl E: Promoter methylation of ID4. A marker for recurrence-free survival in human breast cancer. Pathologe 29 (Suppl 2): S319-S327, 2008 (In German).

19. Yang Q, Shan L, Yoshimura G, Nakamura M, Nakamura Y, Suzuma T, Umemura T, Mori I, Sakurai T and Kakudo K: 5-aza-2'-deoxycytidine induces retinoic acid receptor beta 2 demethylation, cell cycle arrest and growth inhibition in breast carcinoma cells. Anticancer Res 22: 2753-2756, 2002.

20. Wang H, Wang XQ, Xu XP and Lin GW: ID4 methylation predicts high risk of leukemic transformation in patients with myelodysplastic syndrome. Leuk Res 34: 598-604, 2010.

21. Rüter B, Wijermans PW and Lübbert M: DNA methylation as a therapeutic target in hematologic disorders: Recent results in older patients with myelodysplasia and acute myeloid leukemia. Int J Hematol 80: 128-135, 2004.
22. Umetani N, Takeuchi H, Fujimoto A, Shinozaki M, Bilchik AJ and Hoon DS: Epigenetic inactivation of Id4 in colorectal carcinomas correlates with poor differentiation and unfavorable prognosis. Clin Cancer Res 10: 7475-7483, 2004.

23. Vinarskaja A, Goering W, Ingenwerth $M$ and Schulz WA: ID4 is frequently downregulated and partially hypermethylated in prostate cancer. World J Urol 30: 319-325, 2012.

24. Xu RR, Liu F, Cui X, Zhang XW and Wang Y: ID4 promoter methylation in acute myeloid leukemia. J Exp Hematol 19: 582-584, 2011 (In Chinese).

25. Dell'Orso S, Ganci F, Strano S, Blandino G and Fontemaggi G: ID4: A new player in the cancer arena. Oncotarget 1: 48-58, 2010.

26. Chan AS, Tsui WY, Chen X, Chu KM, Chan TL, Chan AS, Li R, So S, Yuen ST and Leung SY: Downregulation of ID4 by promoter hypermethylation in gastric adenocarcinoma. Oncogene 22: 6946-6953, 2003.

27. Qu F, Zhao CH, Diao YQ, Zhu XL, Chen J, Li M, Liu CP, Jiang L and Jin J: Methylation of Id4 gene and inhibitive effect of arsenic trioxide on it in Raji cells. Clin J Hematol 31: 821-825, 2010 (In Chinese).

28. Vandeputte DA, Troost D, Leenstra S, Ijlst-Keizers H, Ramkema M, Bosch DA, Baas F, Das NK and Aronica E: Expression and distribution of id helix-loop-helix proteins in human astrocytic tumors. Glia 38: 329-338, 2002. 